\title{
Diagnostic Performance and Reference Values of Novel Biomarkers of Paediatric Heart
}

\section{Failure}

Jakob A. Hauser ${ }^{1,2,3}$, Svitlana Demyanets ${ }^{4}$, Krisztina Rusai ${ }^{1}$, Clara Goritschan ${ }^{1}$, Michael

Weber $^{5}$, Dilveer Panesar ${ }^{2,3}$, Lisa Rindler ${ }^{1}$, Andrew M. Taylor ${ }^{2,3}$, Rodrig Marculescu ${ }^{4}$, Michael

Burch $^{3}$, Johann Wojta ${ }^{6,7}$, Ina Michel-Behnke ${ }^{1}$

${ }^{1}$ Medical University of Vienna, Department of Paediatrics and Adolescent Medicine,

Division of Paediatric Cardiology; Vienna, Austria

${ }^{2}$ University College London, Institute of Cardiovascular Science, Centre for Cardiovascular Imaging; London, United Kingdom

${ }^{3}$ Great Ormond Street Hospital for Children, Cardiorespiratory Division; London, United Kingdom

${ }^{4}$ Medical University of Vienna, Department of Laboratory Medicine; Vienna, Austria

${ }^{5}$ Medical University of Vienna, Department of Biomedical Imaging and Image-guided

Therapy; Vienna, Austria

${ }^{6}$ Medical University of Vienna, Department of Internal Medicine II, Division of Cardiology;

Vienna, Austria

${ }^{7}$ Medical University of Vienna, Core Facilities; Vienna, Austria 


\section{Corresponding author}

Jakob Hauser

University College London

Centre for Cardiovascular Imaging

30 Guilford Street

London, WC1N 1EH

United Kingdom

Email: jakob.hauser@ucl.ac.uk

Phone: +44 2078138106

Fax: +442078138263

Keywords: heart failure; paediatric; biomarker; cardiomyopathy; congenital heart disease

Word count: 2,978

The Corresponding Author has the right to grant on behalf of all authors and does grant on behalf of all authors, an exclusive license (or non exclusive for government employees) on a worldwide basis to the BMJ Publishing Group Ltd and its Licensees to permit this article (if accepted) to be published in HEART editions and any other BMJPGL products to exploit all subsidiary rights. 


\section{ABSTRACT}

OBECTIVE: Biomarkers play a pivotal role in heart failure (HF) management. Reference values and insights from studies in adults cannot be extrapolated to the paediatric population due to important differences in pathophysiology and compensatory reserve. We assessed the diagnostic utility of four novel biomarkers in paediatric HF.

METHODS: Mid-regional (MR) pro-Atrial Natriuretic Peptide (proANP), sST2, Growth Differentiation Factor-15 (GDF-15), MR-proAdrenomedullin (proADM) and N-terminal proB Natriuretic Peptide (NT-proBNP) were measured in 114 patients and 89 controls. HF was defined as presence of HF symptoms and/or abnormal systolic ventricular function. Receiveroperating characteristics were plotted and the area under the curve (AUC) measured. This was repeated for subgroups with cardiomyopathy and congenital heart disease (CHD). Ventricular systolic function was measured by magnetic resonance or echocardiography. Reference values were calculated according to current guidelines.

RESULTS: The AUC for diagnosing HF was 0.76 for MR-proANP (CI: $0.70-0.84$ ) and 0.82 for NT-proBNP (CI: 0.75-0.88). These parameters performed similarly in the subgroups with CHD and cardiomyopathy. By contrast, MR-proADM, GDF-15 and sST2 performed poorly. When used in conjunction with NT-proBNP, no parameter added significantly to its diagnostic accuracy. NT-proBNP, MR-proANP, GDF-15 and sST2 could accurately discriminate between patients with preserved and patients with poor functional status. In a subset of patients with dilated cardiomyopathy, NT-proBNP, MR-proANP, MR-proADM and GDF-15 were associated with poor LV function.

CONCLUSION: MR-proANP could accurately detect HF in children and adolescents. Its diagnostic performance was comparable to that of NT-proBNP, regardless of the underlying condition. Reference values are presented. 


\section{KEY MESSAGES}

\section{What is already known about this subject?}

In heart failure (HF), blood-based biomarkers provide objective information on diagnosis, prognostic stratification and treatment response. However, validation data and reference values of novel parameters are only available for the adult population, where HF is typically of ischaemic cause and affects the left ventricle. Findings from adult studies cannot be transferred uncritically to the paediatric age group, where important differences in pathophysiology must be considered.

\section{What does this study add?}

The present study shows, for the first time, that MR-proANP can be useful for the diagnosis of HF in children and adolescents. Its accuracy was independent of the underlying HF etiology and was comparable to that of NT-proBNP, the reference standard. By contrast, our findings do not support sufficient diagnostic power for MR-proADM, sST2 and GDF-15. Reference values from normal children are presented.

\section{How might this impact on clinical practice?}

Measuring biomarkers can aid detect HF, grade disease severity and evaluate response to treatment in patients where symptoms are equivocal, which is especially useful in very young children or patients with complex lesions, where imaging studies can be difficult to accomplish accurately. The reference ranges presented in this study will be of use for future research studies and clinical practice. 


\section{INTRODUCTION}

Paediatric heart failure (HF) differs substantially from its adult counterpart. Contrary to the latter, paediatric HF is caused predominantly by the large and heterogeneous group of congenital heart diseases (CHD) and by cardiomyopathy (CMP). Advances in CHD management have led to a rise in prevalence throughout all age groups. ${ }^{1}$ These specifics of paediatric HF present several problems. Symptoms and history are often ambiguous in young children and can be confounded by comorbidities. Functional assessments can be challenging in very small or uncooperative children, in complex CHD or in right ventricular (RV) disease, where echocardiography is unreliable. Measuring biomarkers in the blood can facilitate HF management as they provide objective information on disease severity, prognosis and treatment response..$^{2-6}$

Novel biomarkers typically reflect different components of the complex HF pathophysiology such as fibrotic remodelling, myocardial stretch or inflammation, and their complementary use has been shown to greatly enhance their prognostic and diagnostic power. ${ }^{45-10}$ In adult cardiology, such parameters are usually studied and validated in relatively homogeneous populations of aged and multi-morbid patients with left-sided HF of predominantly ischaemic aetiology. ${ }^{4-9}$ Findings from adults cannot be extrapolated to children as they fail to reflect important differences in pathophysiology and compensatory reserve inherent to this group. We therefore sought to assess four novel biomarkers in paediatric HF patients and to provide reference values from normal children.

\section{METHODS}

\section{Study design and population}

This study was conducted as a prospective trial in two tertiary centres in Austria and the United Kingdom. Patients aged 0-24 years were recruited from a cardiac ward and an HF 
outpatient clinic between September 2013 and July $2015(\mathrm{n}=114)$. This included a subset of patients with dilated CMP (DCM) undergoing elective cardiac magnetic resonance (CMR) imaging $(\mathrm{n}=25)$. HF was defined as presence of HF symptoms and abnormal ventricular systolic function or, in clinically compensated patients without symptoms (i.e. functional class I; see below), history of structural heart disease and abnormal ventricular systolic function on echocardiography or CMR. Abnormal systolic ventricular function was defined as fractional shortening $(\mathrm{FS})<28 \%$ or ejection fraction $(\mathrm{EF})<55 \%$ for the left ventricle $(\mathrm{LV})$ and $\mathrm{EF}<$ $50 \%$ or a Z-score $>2$ for tricuspid annular positive systolic excursion (TAPSE) for the right ventricle (RV). ${ }^{11}$ Patients with prior surgery for CHD were excluded if normal cardiac physiology had been completely restored. Reference values were obtained from children without heart disease undergoing phlebotomy prior to an elective procedure $(n=89)$.

Exclusion criteria were known renal, pulmonary, autoimmune or malignant comorbidity, haemodynamic instability and/or failure to provide informed consent. Written, informed consent was obtained from all patients and their parents prior to inclusion. The study was conducted in conformity with the Declaration of Helsinki and ethical approval was obtained from both institutional review boards.

\section{Functional classification}

Disease severity was graded using the Ross classification. ${ }^{12}$

\section{Biomarker sampling and analysis}

Blood was collected using standard collection techniques on the day of admission in inpatients, or on the day of clinic visit in outpatients. Plasma and serum samples were spun and frozen on the day of collection and stored at $-80^{\circ} \mathrm{C}$ for batch-analysis. Soluble ST2 (sST2; Presage ${ }^{\circledR}$ ST2 Assay, Critical Diagnostics, San Diego, CA, USA) and Growth Differentiation Factor 15 (GDF-15; Human GDF-15 Quantikine ELISA Kit, R\&D Systems, 
Minneapolis, MN, USA) were measured in patient sera using a specific enzyme-linked immunosorbent assays (ELISA). The measurement range was 3.125 to $200 \mathrm{ng} / \mathrm{mL}$ for the Presage ${ }^{\circledR}$ ST2 Assay (based on a 50-fold dilution of patient samples) and 23.40 to 1,500 pg/mL for the Human GDF-15 Quantikine ELISA Kit (based on a 4-fold dilution). An automated immunofluorescent assay (KRYPTOR ${ }^{\circledR}$ System, BRAHMS AG, Hennigsdorf/Berlin, Germany) was used to determine mid-regional pro-Adrenomedullin (MR-proADM) from EDTA-plasma, as well as mid-regional pro-Atrial Natriuretic Peptide (MR-proANP), processed from serum. Measurement ranges were: 2.1 to $10,000.0 \mathrm{pmol} / \mathrm{L}$ for MR-proANP; 0,05 to $100 \mathrm{nmol} / \mathrm{L}$ for MR-proADM. N-terminal pro-B Natriuretic Peptide (NT-proBNP) was assessed using an Elecsys ${ }^{\circledR}$ immunoassay on a Cobas 8000 system (Roche Diagnostics, Mannheim, Germany). Laboratory measurements were performed by investigators who were blinded to patient history.

\section{Imaging protocol}

Using echocardiography, LV diameters and volumes were measured in end-diastole and end-systole as described (Vivid 9; GE Health Care, Fairfield, CT, USA). ${ }^{13}$ CMR was performed on a 1.5 Tesla system using one spine coil and one body matrix coil (Avanto ${ }^{\circledR}$, Siemens AG, Erlangen, Germany). A vectorcardiogram was used for cardiac gating. Ventricular volumes were measured using radial $k$ - $t$ SENSE imaging during free breathing and quantified by manual, slice-wise tracing of the endocardial border in systole and diastole by inclusion of papillary muscles and trabeculae in the myocardium (OsiriX; Pixmeo SARL, Bernex, Switzerland). EF was calculated and Z-scores for LV volumes and diameters obtained from published reference data. ${ }^{13-15}$ 


\section{Statistical analysis}

All statistical analyses were performed by a statistician (M.W.) using the pROC package for R (R Foundation for Statistical Computing, Vienna, Austria) ${ }^{16}$ and SPSS Statistics software version 23.0 (IBM Corp., Armonk, NY, USA). Reference intervals were calculated according to the Clinical and Laboratory Standards Institute (CLSI) guideline C28A3 using MedCalc version 12.5 (MedCalc Software bvba, Ostend, Belgium). ${ }^{17}$ Due to the non-normal distribution and the number of reference values of less than 120 the recommended "robust method" of calculation was applied for all parameters. Metric and normally distributed data are presented as mean \pm standard deviation. In case of skewed data distribution, medians are presented with their interquartile range (IQR). Due to data skewedness in the reference population, associations between biomarker levels and age or functional data were assessed using Spearman's rank correlation. This population was subsequently divided into four age groups and the variance between the biomarker levels of these groups was assessed by Kruskal-Wallis test. In order to assess the association between biomarker levels and LV size and systolic function in patients with DCM, a binary variable was created, whereby LV dysfunction was defined as EF $<55 \%$ or FS $<28 \%$ and LV dilatation as a Z-score $>2$ for LVEDV. The associations between biomarkers and these factors were determined by logistic regression in this subgroup. To examine the accuracy of each blood test to detect HF, a receiver-operating characteristic (ROC) was plotted and the area under the curve (AUC) quantified. 95\% confidence intervals (CI) were obtained from a bootstrap sample of $n=10,000$. This procedure was subsequently repeated in subgroups of patients with CHD and CMP separately. In order to assess the incremental value of using novel parameters in conjunction with NT-proBNP, a step-wise multiple logistic regression model was first built. When a statistically significant association with HF was present, the resulting value from this model was used to repeat the ROC analysis and the diagnostic accuracy of combinations of biomarkers was compared to that of NT-proBNP as a standalone 
parameter according to the method proposed by de Long et al. ${ }^{18}$ A significance level of $p \leq$ 0.05 was defined for all statistical operations.

\section{RESULTS}

\section{Study population}

A total of 203 subjects were enrolled for the study ( 95 female; median age 7.5 years; IQR 2.4 - 13.0; range 5 days to 24 years). This included 114 patients with HF (59 female; median age 5.9 years; IQR 2.1 - 12.5) and 89 controls (36 female; median age 8.9 years; IQR $2.7-14.5)$. No significant differences in sex or age distribution were found between the groups. Clinical data are listed in Table 1. 
Table 1. Study population characteristics.

\begin{tabular}{|l|c|}
\hline Ross / NYHA I and II* & $88(77 \%)$ \\
\hline Ross / NYHA III and IV* & $17(15 \%)$ \\
\hline Dilated cardiomyopathy & $38(33 \%)$ \\
\hline Functional single ventricle & $15(13 \%)$ \\
\hline Pulmonary / right-sided obstruction & $11(10 \%)$ \\
\hline Aortic / left-sided obstruction & $10(9 \%)$ \\
\hline Ventricular septal defect & $9(8 \%)$ \\
\hline Tetralogy of Fallot & $8(7 \%)$ \\
\hline Atrioventricular septal defect & $4(4 \%)$ \\
\hline Patent arterial duct & $4(4 \%)$ \\
\hline Hypertrophic cardiomyopathy & $3(3 \%)$ \\
\hline Restrictive cardiomyopathy & $2(2 \%)$ \\
\hline Atrial septal defect & $2(2 \%)$ \\
\hline Mixed lesion / other & $8(7 \%)$ \\
\hline All variables & \\
\hline
\end{tabular}

All variables listed as $\mathrm{N}(\%)$. *Missing functional class in 9 patients.

\section{Diagnostic accuracy of novel biomarkers in the general study population}

There was a significant difference between groups for NT-proBNP and MR-proANP (Table 2). Similar trends for sST2 and GDF-15 did not reach statistical significance. ROC analysis was undertaken for MR-proANP, sST2, GDF-15 and NT-proBNP in 114 patients and 89 controls (Fig. 1a). Five datasets were due to incomplete data. NT-proBNP and MRproANP showed very good accuracy, whereas the remaining parameters did not perform sufficiently (Table 3). Using an NT-proBNP cut-off value of $94 \mathrm{pg} / \mathrm{mL}$ yielded a sensitivity of $80 \%$ and a specificity of $71 \%$ with a positive predictive value (PPV) of $78 \%$ and a negative 
predictive value (NPV) of 74\%. For MR-proANP, a 48 pmol/L cut-off resulted in a $75 \%$ sensitivity and a specificity of $70 \%$ (PPV $76 \%$ and NPV 68\%). Due to missing data, adding MR-proADM to the head-to-head comparison reduced the number of valid cases to 76 in the HF group and 51 controls (Table 4). ROC analysis showed poor accuracy for MR-proADM, whereas the outcome for the other parameters did not change significantly (Fig. 1b). When comparing their ability to discriminate high from low FC (i.e. I and II vs. III and IV), the AUC for NT-proBNP was 0.95 (CI: $0.90-0.99, p<0.001), 0.93$ for MR-proANP (CI: 0.84 0.99, $p<0.001$ ), 0.89 for GDF-15 (CI: $0.76-0.98, p<0.001)$ and 0.76 for sST2 (CI: $0.57-$ $0.92, p=0.001)$. 
Table 2. Biomarker results in patients, controls and subgroups.

\begin{tabular}{|l|c|c|c|c|}
\hline \multicolumn{1}{|c|}{ Parameter } & Controls $(\mathbf{n}=89)$ & All patients $(\mathbf{n = 1 1 4})$ & Patients with CHD (n=71) & Patients with CMP $(\mathbf{n = 4 3})$ \\
\hline NT-proBNP $(\mathrm{pg} / \mathrm{mL})$ & $66(23-105)^{*}$ & $294(106-893)^{*}$ & $241(105-543)^{*}$ & $408(141-4,758)^{*}$ \\
\hline MR-proANP $(\mathrm{pmol} / \mathrm{L})$ & $40(28-53)^{*}$ & $74.1(47.2-166.7)^{*}$ & $72.8(47.7-172.9)^{*}$ & $76.2(44.7-162.1)^{*}$ \\
\hline sST2 $(\mathrm{ng} / \mathrm{mL})$ & $17.7(13.7-28.8)^{\#}$ & $23.6(14.2-37.3)^{\#}$ & $23.5(13.7-38.1)^{\#}$ & $26.3(15.1-37.5)^{\#}$ \\
\hline GDF-15 $(\mathrm{pg} / \mathrm{mL})$ & $307(241-501)^{\dagger}$ & $405(283-629)^{\dagger}$ & $405(286-591)^{\dagger}$ & $374(277-707)^{\dagger}$ \\
\hline MR-proADM (nmol/L) & $0.34(0.30-0.40)$ & $0.37(0.32-0.48)$ & $0.37(0.32-0.45)$ & $0.37(0.31-0.51)$ \\
\hline
\end{tabular}

Biomarker results for controls, patients with heart failure as well as subgroups with congenital heart disease (CHD) and cardiomyopathy (CMP).

All data are presented as medians with interquartile range. Groups by Kruskal-Wallis test. ${ }^{*} p<0.001 ; \dagger p=0.070 ; \# p=0.068$. NT-proBNP $=$

$\mathrm{N}$-terminal pro-B natriuretic peptide; MR-proANP = mid-regional pro-atrial natriuretic peptide; GDF-15 = Growth differentiating factor 15;

MR-proADM = mid-regional pro-Adrenomedullin. 
Table 3. Receiver-operating analysis for heart failure biomarkers (without MR-proADM)

\begin{tabular}{|c|c|c|c|c|c|c|c|c|c|}
\hline \multirow[t]{2}{*}{ Parameter } & \multicolumn{3}{|c|}{ All patients $(n=114)$} & \multicolumn{3}{|c|}{ Patients with CHD $(n=71)$} & \multicolumn{3}{|c|}{ Patients with CMP $(n=43)$} \\
\hline & $\mathbf{A U C}$ & $95 \% \mathrm{CI}$ & $p$ & AUC & $95 \% \mathrm{CI}$ & $p$ & AUC & $95 \% \mathrm{CI}$ & $p$ \\
\hline NT-proBNP & 0.82 & $0.75-0.88$ & $<0.001$ & 0.81 & $0.72-0.88$ & $<0.001$ & 0.84 & $0.75-0.92$ & $<0.001$ \\
\hline MR-proANP & 0.76 & $0.70-0.84$ & $<0.001$ & 0.76 & $0.69-0.86$ & $<0.001$ & 0.76 & $0.66-0.86$ & $<0.001$ \\
\hline sST2 & 0.60 & $0.52-0.69$ & $<0.05$ & 0.59 & $0.53-0.72$ & 0.051 & 0.60 & $0.47-0.69$ & 0.063 \\
\hline GDF-15 & 0.58 & $0.50-0.67$ & 0.051 & 0.58 & $0.48-0.68$ & 0.104 & 0.59 & $0.47-0.69$ & 0.101 \\
\hline NT-proBNP + GDF-15 & 0.82 & $0.75-0.88$ & 1.000 & 0.80 & $0.72-0.88$ & 1.000 & 0.84 & $0.75-0.92$ & 1.000 \\
\hline MR-proANP + GDF-15 & 0.80 & $0.73-0.86$ & 0.585 & 0.80 & $0.71-0.87$ & 0.887 & 0.81 & $0.71-0.89$ & 0.399 \\
\hline $\begin{array}{l}\text { NT-proBNP + } \\
\text { MRproANP + GDF-15 }\end{array}$ & 0.84 & $0.78-0.90$ & 0.359 & 0.83 & $0.76-0.90$ & 0.372 & 0.81 & $0.71-0.89$ & 0.399 \\
\hline
\end{tabular}

Diagnostic performance of biomarkers for the diagnosis of heart failure (HF) in all patients and subgroups with congenital heart disease

(CHD) and cardiomyopathy (CMP). A receiver-operating characteristic was plotted and the area under the curve (AUC) measured in order to compare accuracies between tests. $95 \%$ confidence intervals $(\mathrm{CI})$ were obtained from a bootstrap sample of $n=10,000$. NT-proBNP $=\mathrm{N}$ terminal pro-B natriuretic peptide; MR-proANP = mid-regional pro-atrial natriuretic peptide; GDF-15 = Growth differentiating factor 15; MR-proADM = mid-regional pro-Adrenomedullin. 
Table 4. Receiver-operating analysis for heart failure biomarkers (with MR-proADM).

\begin{tabular}{|c|c|c|c|c|c|c|c|c|c|}
\hline \multirow[t]{2}{*}{ Parameter } & \multicolumn{3}{|c|}{ All patients $(n=114)$} & \multicolumn{3}{|c|}{ Patients with CHD $(n=71)$} & \multicolumn{3}{|c|}{ Patients with CMP $(n=43)$} \\
\hline & AUC & $95 \% \mathrm{CI}$ & $p$ & AUC & $95 \% \mathrm{CI}$ & $p$ & $\mathbf{A U C}$ & $95 \% \mathrm{CI}$ & $p$ \\
\hline NT-proBNP & 0.84 & $0.76-0.88$ & $<0.001$ & 0.84 & $0.75-0.88$ & $<0.001$ & 0.83 & $0.76-0.91$ & $<0.001$ \\
\hline MR-proANP & 0.80 & $0.70-0.84$ & $<0.001$ & 0.81 & $0.69-0.84$ & $<0.001$ & 0.79 & $0.66-0.85$ & $<0.001$ \\
\hline sST2 & 0.60 & $0.52-0.69$ & 0.058 & 0.56 & $0.50-0.68$ & 0.328 & 0.64 & $0.49-0.71$ & 0.026 \\
\hline GDF-15 & 0.57 & $0.51-0.68$ & 0.204 & 0.57 & $0.50-0.68$ & 0.249 & 0.56 & $0.50-0.68$ & 0.324 \\
\hline MR-proADM & 0.61 & $0.48-0.69$ & 0.029 & 0.62 & $0.50-0.73$ & 0.066 & 0.61 & $0.49-0.73$ & 0.066 \\
\hline
\end{tabular}

Diagnostic performance of biomarkers for the diagnosis of heart failure (HF) in all patients and subgroups with congenital heart disease (CHD) and cardiomyopathy (CMP). A receiver-operating characteristic was plotted and the area under the curve (AUC) measured in order to compare accuracies between tests. $95 \%$ confidence intervals $(\mathrm{CI})$ were obtained from a bootstrap sample of $n=10,000$. NT-proBNP $=\mathrm{N}$ terminal pro-B natriuretic peptide; MR-proANP = mid-regional pro-atrial natriuretic peptide; GDF-15 = Growth differentiating factor 15 ; MR-proADM = mid-regional pro-Adrenomedullin. 


\section{Diagnostic accuracy in congenital heart disease}

Biomarker levels and age did not differ significantly between patients with univentricular and biventricular physiology (Table 5). NT-proBNP and MR-proANP displayed good accuracy in detecting HF, whereas the other parameters performed poorly (Table 3; Fig. 1d). Using an NT-proBNP cut-off value of $94 \mathrm{pg} / \mathrm{mL}$ yielded a sensitivity of $82 \%$ and a specificity of $70 \%$, giving a PPV of $68 \%$ and a NPV of $83 \%$. For MR-proANP, a $48 \mathrm{pmol} / \mathrm{L}$ cut-off resulted in a 75\% sensitivity and a specificity of 71\% (PPV 67\% and NPV $78 \%$ ). Adding MR-proADM to the analysis reduced the number of valid cases to 38 in the HF group and 51 controls (Table 4). This revealed an unsatisfactory diagnostic power for MRproADM, while that of the other parameters did not change significantly (Fig. 1e). 
Table 5. Biomarker data and surgical history of CHD patients.

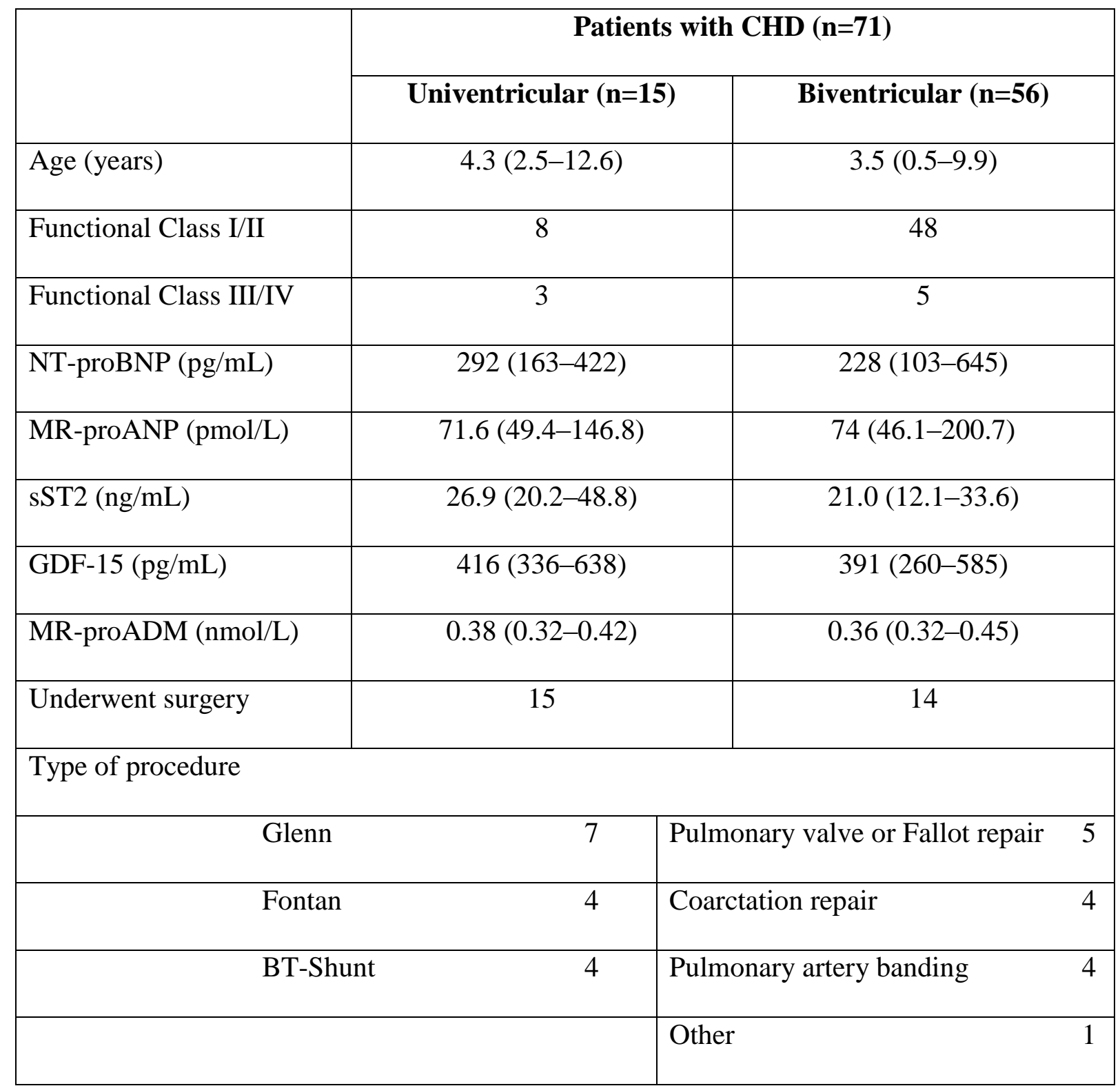

Age and biomarker levels did not differ significantly between patients with univentricular and biventricular physiology $(p>0.05)$ NT-proBNP $=\mathrm{N}$-terminal pro-B natriuretic peptide; MR-proANP = mid-regional pro-atrial natriuretic peptide; GDF-15 = Growth differentiating factor 15; MR-proADM = mid-regional pro-Adrenomedullin. 


\section{Diagnostic accuracy in cardiomyopathy}

In the CMP subgroup, ROC analysis showed good performance for NT-proBNP and MR-proANP (Table 3, Fig. 1g) but not for the remaining parameters. The addition of MRproADM reduced the case number to 38 patients and showed poor accuracy for MRproADM, whereas the performance of the other parameters did not change significantly (Table 4, Fig. 1h). Using an NT-proBNP cut-off value of $134 \mathrm{pg} / \mathrm{mL}$ yielded a sensitivity of $77 \%$ and a specificity of $82 \%$ (PPV 67\% and NPV 88\%). For MR-proANP, a 51 pmol/L cutoff resulted in $74 \%$ sensitivity and a specificity of 74\% (PPV 58\% and NPV 86\%).

In 25 DCM patients with available CMR data, significant correlation of LV EF was found with NT-proBNP $(\mathrm{r}=-0.59, p<0.01)$, MR-proANP $(\mathrm{r}=-0.67, p<0.001)$, GDF-15 ( $\mathrm{r}$ $=-0.62, p<0.001)$ and MR-proADM $(\mathrm{r}=-0.45, p<0.05)$, whereas correlation with sST2 failed to reach statistical significance $(r=-0.29, p=0.167)$. Significant correlation with LVEDV was found for NT-proBNP $(r=0.50, p<0.05)$ and MR-proANP $(r=0.46, p<0.05)$. In the regression model of all 38 DCM patients with available imaging data, only NT-proBNP was predictive of $\mathrm{LV}$ dilatation $(\mathrm{B}=0.003, \mathrm{CI}: 0.002-0.005, p<0.05)$. This was independent of age. Moreover, age-adjusted NT-proBNP and sST2 were predictive of LV dysfunction (NT-proBNP: B =0.001, CI: $0.0001-0.002, p<0.05$; sST2: $\mathrm{B}=0.003$; CI: $0.0001-0.006, p<0.05)$. When removed from the model, higher age remained an independent predictor for poor LV function ( $\mathrm{B}=0.10, \mathrm{CI}: 0.014-0.19, p<0.05)$.

\section{Combined use of HF biomarkers}

In the regression model, the combinations of NT-proBNP and GDF-15, MR-proANP and GDF-15 as well as that of all three parameters were good predictors of HF. However, no combination yielded a significant increment in AUC over NT-proBNP as a standalone parameter (Table 3; Fig. 1c, 1f and 1i). 


\section{Reference values and association with sex and age}

Reference values are presented in Table 6. Significant correlation with age was found for $\operatorname{sST} 2(\mathrm{r}=0.278, p<0.01)$ and MR-proANP $(\mathrm{r}=-0.221, p<0.05)$. Moreover, there were trends for GDF-15 $(\mathrm{r}=-0.192, p=0.072)$ and NT-proBNP $(\mathrm{r}=-0.205, p=0.055)$. NTproBNP and MR-proANP showed peaks in the first year, whereas GDF-15 and MR-proADM were highest in children aged six years and younger (Table 7). By contrast, sST2 was highest in the age group of twelve years and older. Females had significantly higher levels of sST2 and MR-proANP, though for the latter, this difference was only minor. No differences between sexes were found for the other blood parameters.

Table 6. Reference values from healthy children.

\begin{tabular}{|l|c|c|c|c|}
\hline Parameter & $\mathrm{N}$ & $\begin{array}{c}\text { 95\% Reference } \\
\text { Interval }\end{array}$ & $\begin{array}{c}\text { Lower Limit } \\
90 \% \mathrm{CI}\end{array}$ & $\begin{array}{c}\text { Upper Limit } \\
90 \% \text { CI }\end{array}$ \\
\hline NT-proBNP $(\mathrm{pg} / \mathrm{mL})$ & 88 & $<258$ & - & $178-326$ \\
\hline MR-proANP $(\mathrm{pmol} / \mathrm{L}($ & 88 & $<115.9$ & - & $82.4-144.8$ \\
\hline sST2 $(\mathrm{pg} / \mathrm{mL})$ & 89 & $<44.4$ & - & $38.2-50.0$ \\
\hline GDF-15 $(\mathrm{pg} / \mathrm{mL})$ & 89 & $<1,736$ & - & $1,059-2,269$ \\
\hline MR-proADM (nmol/L) & 52 & $0.16-0.53$ & $0.12-0.20$ & $0.47-0.57$ \\
\hline
\end{tabular}

Reference intervals were calculated according to the Clinical and Laboratory

Standards Institute (CLSI) guideline C28-A3. Due to the non-normal distribution and the number of reference values of less than 120 the recommended "robust method" of calculation was applied for all parameters. 
Table 7. Values from normal children and association with age and sex.

\begin{tabular}{|c|c|c|c|c|}
\hline Parameter & $\mathbf{N}$ & Mean & Standard deviation & $p$-value \\
\hline NT-proBNP (pg/mL) & 88 & 103.1 & 158.9 & \\
\hline Female & 36 & 81.2 & 119.3 & \multirow{2}{*}{$0.104^{\#}$} \\
\hline Male & 52 & 118.2 & 180.9 & \\
\hline$<1$ year & 10 & 229.4 & 250.0 & \multirow{4}{*}{$<0.001^{\dagger}$} \\
\hline $1-6$ years & 23 & 105.4 & 72.4 & \\
\hline $6-12$ years & 24 & 82.3 & 77.2 & \\
\hline$>12$ years & 31 & 76.6 & 199.2 & \\
\hline MR-proANP (pmol/L) & 88 & 49.6 & 46.7 & \\
\hline Female & 35 & 48.3 & 43.9 & \multirow{2}{*}{$0.008^{\#}$} \\
\hline Male & 53 & 50.5 & 48.8 & \\
\hline$<1$ year & 10 & 78.0 & 68.5 & \multirow{4}{*}{$<0.001^{\dagger}$} \\
\hline $1-6$ years & 24 & 59.2 & 31.9 & \\
\hline $6-12$ years & 24 & 34.5 & 19.8 & \\
\hline$>12$ years & 30 & 44.6 & 58.2 & \\
\hline sST2 (ng/mL) & 89 & 22.4 & 14.9 & \\
\hline Female & 36 & 26.3 & 16.9 & \multirow{2}{*}{$0.042^{\#}$} \\
\hline Male & 53 & 19.8 & 12.8 & \\
\hline$<1$ year & 10 & 18.6 & 10.2 & \multirow{4}{*}{$0.228^{\dagger}$} \\
\hline $1-6$ years & 24 & 19.9 & 12.0 & \\
\hline $6-12$ years & 24 & 18.7 & 12.7 & \\
\hline$>12$ years & 31 & 28.6 & 18.0 & \\
\hline GDF-15 (pg/mL) & 89 & 572.4 & 851.3 & \\
\hline
\end{tabular}




\begin{tabular}{|c|c|c|c|c|}
\hline Female & 36 & 567.1 & 886.4 & \multirow{2}{*}{$0.593^{\#}$} \\
\hline Male & 53 & 575.9 & 835.1 & \\
\hline$<1$ year & 10 & 661.1 & 393.9 & \multirow{4}{*}{$<0.001^{\dagger}$} \\
\hline 1 - 6 years & 24 & 917.2 & $1,197.4$ & \\
\hline $6-12$ years & 24 & 286.1 & 163.4 & \\
\hline$>12$ years & 31 & 498.4 & 889.1 & \\
\hline MR-proADM (nmol/L) & 52 & 0.35 & 0.09 & \\
\hline Female & 21 & 0.33 & 0.09 & \multirow{2}{*}{$0.445^{\#}$} \\
\hline Male & 31 & 0.36 & 0.09 & \\
\hline $0-6$ years & 18 & 0.39 & 0.10 & \multirow{3}{*}{$0.167^{\dagger}$} \\
\hline $6-12$ years & 17 & 0.32 & 0.09 & \\
\hline$>12$ years & 17 & 0.34 & 0.07 & \\
\hline
\end{tabular}

$\dagger$ Assessed by Kruskal-Willis test and \# by Mann-Whitney-U test.

NT-proBNP $=$ N-terminal pro-B natriuretic peptide MR-proANP $=$ mid-regional proatrial natriuretic peptide; GDF-15 = Growth differentiating factor 15; MR-proADM = mid-regional pro-Adrenomedullin.

\section{DISCUSSION}

The present study is the first to demonstrate the diagnostic utility of MR-proANP in the diagnosis of paediatric HF, irrespective of the underlying cause. By contrast, MRproADM, GDF-15, and sST2 did not perform sufficiently, and even when used in conjunction with the reference standard, NT-proBNP, none of the novel parameters added significantly to its diagnostic properties. 
Only two studies have previously addressed the potential role of MR-proANP in paediatric cardiovascular disease. ${ }^{319}$ In adults, it was shown to be superior to NT-proBNP in the diagnosis and prognostic stratification of HF in elderly and overweight patients, where NT-proBNP is less reliable. ${ }^{47}$ In our study, the performance of NT-proBNP was slightly superior to that of MR-proANP and our findings underpin previous work that demonstrated good diagnostic accuracy in a broad spectrum of cardiovascular abnormalities of the paediatric age. ${ }^{26}$ Increases in MR-proANP and NT-proBNP are attributed to closely related physiological mechanisms and the causes underlying the disparity in their diagnostic power can only be speculated about. MR-proANP is typically elevated as a consequence of raised atrial wall stretch, whereas NT-proBNP increases predominantly in response to ventricular wall stretch. ${ }^{20}$ Moreover, cytokines have been found to influence NT-proBNP expression and secretion, while that of MR-proANP appears to be unaffected. ${ }^{21}$ Age-specific differences in filling pressures, atrial wall stretch and cytokine activity could account for the differences in the diagnostic performance of these two parameters observed in our study.

Adult HF is predominantly caused by ischaemic heart disease, a condition typically associated with a chronic state of systemic inflammation and an endpoint of fibrotic remodelling of the myocardium. ${ }^{5}$ In children, where CHD is the leading cause, the importance and prevalence of such fibrotic processes in different lesions is still poorly understood. This may partly explain the lack of diagnostic performance observed for sST2, one of the pivotal developments in HF biomarkers. Its clinical utility is well established both as a standalone parameter and in combination with NT-proBNP, in acute and chronic HF as well as in CAD. ${ }^{8-}$ 102223 In children, it was found to be elevated in the setting of acute Kawasaki disease where it was associated with poor diastolic function. However, sST2 was measured in the context of acute inflammation and $\mathrm{CAD}$, which limits inferences for paediatric $\mathrm{HF}$ and underpins that inflammation is an important factor in its biology. ${ }^{24}$ 
In adults with CHD, GDF-15 was recently shown to be independently associated with elevated pulmonary pressures, poor functional status and cardiac dysfunction. ${ }^{25} 26$ By contrast, paediatric data are sparse, particularly in patients with CMP. In a mixed population of both children and adults with Fontan circulation, one group found elevated GDF-15 levels to be associated with poor functional status and impaired haemodynamic function. However, their findings cannot be uncritically transferred to the general paediatric HF population, which is predominantly biventricular in physiology.${ }^{27}$ Similar data from Fontan patients were published for MR-proADM. ${ }^{28}$ Our findings are partly in keeping with the work of Norozi et al. who found GDF-15 to be associated with low FC and poor function in patients with CHD. ${ }^{25}$ However, their patients were predominantly adults and no effort was undertaken to provide details on comorbidities that potentially affect GDF-15 levels (e.g. renal disease). Moreover, the authors excluded CMP from the analysis and did not provide reference data from healthy controls. The ability of GDF-15 to distinguish between HF patients and controls was poor in our population, even though an association with impaired cardiac function was observed in the DCM group, as it was with MR-proADM. GDF-15 and MR-proADM can be elevated in various conditions and are expressed by different tissues. In cardiomyocytes, GDF-15 is secreted in the presence of abnormal loading conditions and metabolic stress, such as ischaemia, whereas MR-proADM is produced in response to myocardial stretch among other causes. ${ }^{5}$. Notably, in our study levels of both parameters were significantly lower than previously described in adults. ${ }^{79}$ Age-related disparities in compensatory mechanisms, medication, comorbidity, cardiac loading conditions and underlying pathology are conceivable explanations for this phenomenon.

While paediatric reference values have been published recently for GDF-15 and sST2, such normal data for MR-proANP and MR-proADM outside of the neonatal period are 
largely unavailable. ${ }^{19} 2930$ In our reference population, MR-proANP was significantly higher in the first year of life, in keeping with previous studies on B-type natriuretic peptides. ${ }^{6}$ Our finding that sST2 levels were higher in females and in older children is inconsistent with recently published data from a larger cohort. ${ }^{23} 30$ As our reference data are overall consistent with published work this finding is likely to be incidental in nature.

\section{Limitations}

The inhomogeneity of our cohort in terms of underlying aetiology as well as the relatively small proportion of patients with severe HF may have obfuscated some of the statistical associations present in specific conditions and did not allow for a meaningful analysis of the associations between biomarkers and cardiac function. For this reason, we performed a subgroup analysis in a homogeneous group of DCM patients. Moreover, a longitudinal analysis would have been problematic to interpret as outcome trajectories vary substantially between different diseases in the paediatric age group. Further research is required to investigate whether and how reliably these novel biomarkers can predict outcome or treatment response in a more narrowly defined population. Finally, larger case numbers are needed to generate reliable reference intervals for specific age groups and in particular, our sample size may have been underpowered for MR-proADM.

\section{Conclusion}

MR-proANP can accurately detect HF in children and adolescents with CHD and CMP, even if well compensated. Its diagnostic performance was comparable to that of NTproBNP, regardless of the underlying condition. Further research is warranted to evaluate the diagnostic and prognostic significance of these tests in specific paediatric cardiac diseases.

CONFLICT OF INTEREST: none declared 
FUNDING: This work was supported by the Austrian Society of Cardiology (personal grant to J.H. with funds to M.W.) and the European Commission (funds to J.H. and D.P.) as part of the MD-PAEDIGREE research project (contract no. 600932).

\section{REFERENCES}

1. Hsu DT, Pearson GD. Heart failure in children: part I: history, etiology, and pathophysiology. Circ Heart Fail 2009;2(1):63-70.

2. Mangat J, Carter C, Riley G, et al. The clinical utility of brain natriuretic peptide in paediatric left ventricular failure. Eur J Heart Fail 2009;11(1):48-52.

3. Heise G, Lemmer J, Weng Y, et al. Biomarker responses during mid-term mechanical cardiac support in children. J Heart Lung Transplant 2008;27(2):150-7.

4. von Haehling S, Jankowska EA, Morgenthaler NG, et al. Comparison of midregional proatrial natriuretic peptide with $\mathrm{N}$-terminal pro-B-type natriuretic peptide in predicting survival in patients with chronic heart failure. J Am Coll Cardiol 2007;50(20):197380.

5. Gaggin HK, Januzzi JL, Jr. Biomarkers and diagnostics in heart failure. Biochim Biophys Acta 2013;1832(12):2442-50.

6. Cantinotti M, Law Y, Vittorini S, et al. The potential and limitations of plasma BNP measurement in the diagnosis, prognosis, and management of children with heart failure due to congenital cardiac disease: an update. Heart Fail Rev 2014;19(6):72742.

7. Lassus J, Gayat E, Mueller C, et al. Incremental value of biomarkers to clinical variables for mortality prediction in acutely decompensated heart failure: the Multinational Observational Cohort on Acute Heart Failure (MOCA) study. Int J Cardiol 2013;168(3):2186-94. 
8. Bayes-Genis A, de Antonio M, Galan A, et al. Combined use of high-sensitivity ST2 and NTproBNP to improve the prediction of death in heart failure. Eur J Heart Fail 2012;14(1):32-8.

9. Santhanakrishnan R, Chong JP, Ng TP, et al. Growth differentiation factor 15, ST2, highsensitivity troponin $\mathrm{T}$, and $\mathrm{N}$-terminal pro brain natriuretic peptide in heart failure with preserved vs. reduced ejection fraction. Eur J Heart Fail 2012;14(12):1338-47.

10. Sabatine MS, Morrow DA, Higgins LJ, et al. Complementary roles for biomarkers of biomechanical strain ST2 and N-terminal prohormone B-type natriuretic peptide in patients with ST-elevation myocardial infarction. Circulation 2008;117(15):1936-44.

11. Koestenberger M, Ravekes W, Everett AD, et al. Right ventricular function in infants, children and adolescents: reference values of the tricuspid annular plane systolic excursion (TAPSE) in 640 healthy patients and calculation of $\mathrm{z}$ score values. J Am Soc Echocardiogr 2009;22(6):715-9.

12. Ross RD. The Ross classification for heart failure in children after 25 years: a review and an age-stratified revision. Pediatr Cardiol 2012;33(8):1295-300.

13. Lang RM, Bierig M, Devereux RB, et al. Recommendations for chamber quantification: a report from the American Society of Echocardiography's Guidelines and Standards Committee and the Chamber Quantification Writing Group, developed in conjunction with the European Association of Echocardiography, a branch of the European Society of Cardiology. J Am Soc Echocardiogr 2005;18(12):1440-63.

14. Robbers-Visser D, Boersma E, Helbing WA. Normal biventricular function, volumes, and mass in children aged 8 to 17 years. J Magn Reson Imaging 2009;29(3):552-9.

15. Kampmann C, Wiethoff CM, Wenzel A, et al. Normal values of M mode echocardiographic measurements of more than 2000 healthy infants and children in central Europe. Heart 2000;83(6):667-72. 
16. Robin X, Turck N, Hainard A, et al. pROC: an open-source package for R and S+ to analyze and compare ROC curves. BMC Bioinformatics 2011;12:77.

17. Clinical and Laboratory Standards Institute. CLSI. Defining, Establishing, and Verifying Reference Intervals in the Clinical Laboratory. Third ed. Wayne, Pa.: Clinical and Laboratory Standards Institute, 2008.

18. DeLong ER, DeLong DM, Clarke-Pearson DL. Comparing the areas under two or more correlated receiver operating characteristic curves: a nonparametric approach. Biometrics 1988;44(3):837-45.

19. Grass B, Baumann P, Arlettaz R, et al. Cardiovascular biomarkers pro-atrial natriuretic peptide and pro-endothelin-1 to monitor ductus arteriosus evolution in very preterm infants. Early Hum Dev 2014;90(6):293-8.

20. Yasue H, Yoshimura M, Sumida H, et al. Localization and mechanism of secretion of Btype natriuretic peptide in comparison with those of A-type natriuretic peptide in normal subjects and patients with heart failure. Circulation 1994;90(1):195-203.

21. McGrath MF, de Bold ML, de Bold AJ. The endocrine function of the heart. Trends Endocrinol Metab 2005;16(10):469-77.

22. Weinberg EO, Shimpo M, Hurwitz S, et al. Identification of serum soluble ST2 receptor as a novel heart failure biomarker. Circulation 2003;107(5):721-6.

23. Dieplinger B, Januzzi JL, Jr., Steinmair M, et al. Analytical and clinical evaluation of a novel high-sensitivity assay for measurement of soluble ST2 in human plasma--the Presage ST2 assay. Clin Chim Acta 2009;409(1-2):33-40.

24. Sato YZ, Molkara DP, Daniels LB, et al. Cardiovascular biomarkers in acute Kawasaki disease. Int J Cardiol 2013;164(1):58-63.

25. Norozi K, Buchhorn R, Yasin A, et al. Growth differentiation factor 15: an additional diagnostic tool for the risk stratification of developing heart failure in patients with operated congenital heart defects? Am Heart J 2011;162(1):131-5. 
26. Eindhoven JA, van den Bosch AE, Oemrawsingh RM, et al. Release of growthdifferentiation factor 15 and associations with cardiac function in adult patients with congenital heart disease. Int J Cardiol 2016;202:246-51.

27. Raedle-Hurst TM, Koenigstein K, Gruenhage F, et al. Growth differentiation factor 15--an early marker of abnormal function of the Fontan circuit in patients with univentricular hearts. Am Heart J 2010;160(6):1105-12.

28. Kaiser R, Abdul-Khaliq H, Wilkens H, et al. Mid-regional pro-adrenomedullin: an indicator of the failing Fontan circuit in patients with univentricular hearts? Eur J Heart Fail 2014;16(10):1082-8.

29. Koch L, Dabek MT, Frommhold D, et al. Stable precursor fragments of vasoactive peptides in umbilical cord blood of term and preterm infants. Horm Res Paediatr 2011;76(4):234-9.

30. Meeusen JW, Johnson JN, Gray A, et al. Soluble ST2 and galectin-3 in pediatric patients without heart failure. Clin Biochem 2015. 